\title{
POR UN ANALISIS SEMIOTICO DEL CURRICULUM
}

\section{INTRODUCCION}

\author{
JOSÉ LUIS RODRÍGUEZ ILLERA \\ Universidad Central de Barcelona
}

La idea de que el currículum puede ser efectivamente estudiado, de que constituye uno de los principales temas de estudio de la pedagogía (desde la propia teoría de la educación, hasta especialmente la didáctica) conlleva algún tipo de presuposición sobre el tipo de concepto, objeto, sistema o constructo que el curriculum sea. Pues es obvio que no cualquier tema ocupa ese lugar central que el curriculum va cada día adquiriendo, y es obvio también que su realidad dista mucho de esa epistemología ingenua que asocia realidad sólo a lo más estrictamente empírico. Al contrario, el currículum es siempre una realidad construida, y ello en el doble sentido siguiente: construida en cuanto a su elaboración, pues constituye una selección de la cultura, aquella que se considera que debe ser transmitida; realidad construida también como objeto de la teoría, como tema de reflexión, como realidad que se nos hace a través del discurso teórico.

Pensar, pues, el curriculum es algo más que simplemente hablar sobre él, como si fuese un dato ya dado, algo que existe por sí mismo. Esta confusión proviene de un empirismo ingenuo, de un empirismo que se fortifica en los hechos, como si los hechos existiesen independientemente del observador. $\mathrm{Y}$ el hecho del currículum, lo que supuestamente lo convierte en objeto real, es el uso lingüístico que del témino se hace cuando se habla de educación a un nivel "social" o/y "organizativo" 1 . Así, por ejemplo, los denominados decretos de mínimos pueden ser pensados perfectamente como la plasmación "política" del currículum: la parte mínima de la cultura que el Estado obliga a transmitir; o, también, las nuevas asignaturas o actividades que una escuela programa para un determinado curso pueden pensarse como el currículum que esa escuela elabora dentro de los límites de autonomía que una determinada institución educativa tiene. Si se conviene en lo habitual de ambos ejemplos, se convendrá también en que en ambos casos el término currículum se utiliza para dar cuenta de lo que genéricamente se denominan los contenidos escolares: lo que el maestro tiene

1. La cuestión de los niveles de uso del término educación, la hemos tratado con algún detalle anteriormente (Rodríguez Illera, 1982, y ligeramente retocada: 1985), por lo que no volveremos a insistir aquí sobre este punto. 
que enseñar, o, a la inversa, lo que el alumno tiene que aprender. Sin embargo, nada más falso: lo que está en juego en la relación educativa -en cualquier relación educativa- es siempre mucho más que una simple transmisión de contenidos, como por otra parte siempre han reconocido todos los pedagogos (ya intentaremos retomar este punto más adelante). Por tanto, en muchos casos, el concepto de currículum construye una versión idealizada - por simplificada - de la relación educativa. Nosotros intentaremos pensarlo en toda la complejidad que suponemos que tiene, utilizando en especial, aunque no exclusivamente, conceptos poco habituales en teoría de la educación, provinientes fundamentalmente de la semiótica (conceptos como: esquema narrativo, modalidades, actantes, etc.), y que esperamos que nos permitan aclarar algunos aspectos de esta amplia problemática.

Para ello, intentaremos aproximarnos ordenadamente a un conjunto de problemas, por mas que estén mutuamente presupuestos y en ocasiones nos resulte imposible seguir un decurso lineal.

\section{CURRICULUM Y METACOMUNICACION}

(1) Empezaremos señalando lo que podríamos denominar la "formalidad" del currículum, por parecernos lo más obvio: el término sólo puede designar, cuando se utiliza teóricamente, una estructura capaz de subsumir los múltiples currícula-ocurrencia. Si nos atuviésemos a éstos resultaría en exceso difícil poder pensarlos sincrónicamente, formando una estructura: sólo nos resultarían visibles sus aspectos diferentes e históricos. Se comprenderá que intentamos fundar nuestra aproximación en el concepto sausureano es extremadamente general y que, quizás por ello mismo, ha inspirado numerosas analogías en las ciencias sociales y humanas a lo largo de este siglo.

Por tanto, al igual que la lengua, el currículum debe considerarse sólo como forma (opuesta aquí a sustancia, según la terminología de Hjemslev), y dejando las relaciones entre ambas fuera de nuestro estudio. Esto conlleva grandes consecuencias para la manera de abordar el problema: enfatizar el estudio sincrónico; buscar sus unidades significativas, los signos, si pretendemos movernos en el curso histórico de la analogía que no será el caso); plantear la cuestión del valor, esto es, una definición relacional (y diferencial) de los signos; distinguir un eje paradigmático de uno sintagmático, o un plano de la selección (de los signos) y otro de su combinación - según la lectura y terminología de Jakobson. Todas estas premisas metodológicas, y otras más específicas de la semiótica narrativa, pueden ser un marco en el que movernos para intentar sistematizar muchas cuestiones -teóricassobre el currículum. Pero también es cierto que la lingüística saussureana adolece de un buen número de problemas; entre ellos, el no poder cuestio- 
narse el contexto social en el que se da el lenguaje (para nuestro caso, el currículum ${ }^{2}$ ).

(2) En segundo lugar, y entrando ya en el tema, quisiéramos discutir la cuestión conceptual de la distinción entre currículum y currículum oculto. Como con ningún otro concepto se ha planteado aquí una escisión tan fundamental, a la vez que tan confusa. Currículum oculto parece querer referirse - pues, entre otras cosas, el referente de la expresión no es claro- a alguna de las siguientes posibilidades:

a) a lo que efectivamente no se transmite.

b) a lo que se transmite sin saberlo ni quererlo por parte del enseñante.

c) a lo que se quiere transmitir sin explicitarlo.

d) a la "ideología".

Estas posibilidades son muy diferentes. Por ejemplo, lo que no se transmite es, simplemente, lo que se excluye del campo de lo enseñable -aunque ya veremos las dificultades que entraña definirlo. Lo mismo ocurre con el caso siguiente: se acude a la conciencia del enseñante, pero es más amplio: muchas definiciones de currículum oculto inciden en la idea de lo que la propia organización escolar transmite por su mera existencia, etc.

Podríamos haber buscado otras alternativas, pero casi todas o todas las definiciones habituales del currículum oculto caen en alguna de estas posibilidades (para un análisis histórico y conceptual, Vázquez 1983) ${ }^{3}$. Por otra parte son suficientes para comprobar las distintas pertinencias que las fundamentan: por una parte, la escisión entre enseñar y transmitir (se transmite más de lo que se enseña, en caso contrario no habría lugar para el concepto de currículum oculto); por otra, la oposición implícito/explicito e incluso también la oposición denotación/connotación (por ejemplo, la ideología como lo connotado). Este juego de oposiciones remite a una más general, que de alguna manera las fundamenta pero que tampoco es nítida: la que separa, junta u opone -según los casos-comunicación y metacomunicación. El ùltimo concepto recubre un fenómeno habitual: el lenguaje y la comunicación en general dicen o comunican más de lo dicho, más del

2. Conviene quizás recordar que no todas las lecturas de Saussure comparten este punto de vista "estándard". Por ejemplo, Pêcheux (1969) cree que la definición de la lengua como institución social en el Cours permite el planteamiento de las condiciones (sociales) de producción de un/el discurso. Por otra parte, toda la sociolingüistica se constituye por la negación explícita de esta actitud "neutral" del enfoque saussurreano (por ejemplo, Aracil, 1982, Bourdieu 1982).

3. El artículo citado de Vázquez contiene la bibliografía principal sobre el tema. Algún trabajo importante se encuentra en castellano, por ejemplo en la compilación de Gimeno Sacristan y Pérez Gómez (1983). También en Aronowitz y Giroux (1985) se encuentran reflexiones importantes sobre el tema, así como muy especialmente en otros trabajos del propio Giroux. 
contenido estricto del mensaje $\mathrm{e}^{4}$ Las correspondencias podrían ser aproximadamente como sigue:

comunicación

metacomunicación

enseñar

transmitir currículum

currículum oculto denotación

connotación

Aunque esta derivación sea ilegítima ( $y$ aparezcan como semejantes comunicación y denotación, o metacomunicación y connotación, mezclando conceptos de diferente nivel de realidad semiótica), sólo es así si se piensa en ambas líneas como recorridos distintos y estancos. Dicho de otra forma, denotación y connotación son dos aspectos del proceso de significación inseparables; lo mismo ocurre con comunicación y metacomunicación, o con la pareja explícito/implícito y también con enseñar/transmitir. Se trata, por emplear una analogía célebre, de algo parecido a las dos superficies que delimita una banda de Moebius: son diferentes, pero el paso de una a otra se realiza sin solución de continuidad.

Currículum y currículum oculto se nos aparecen, siguiendo este hilo de razonamiento, como extremos de un único continuum más que como estructuras encajadas una en otra, que podemos ordenar según varios criterios distintos: según un criterio de visibilidad,por ejemplo, el currículum sólo sería la parte manifiesta, más explícita, de un proceso de transmisión mucho más amplio (el curriculum oculto); según un criterio de intencionalidad, el currículum sería el conjunto de contenidos seleccionados y enseñados intencionalmente; según un criterio "ideológico", el currículum constituiría la ideología "explícita" mientras que el currículum oculto conformaría la ideología "implícita" (incluso para los propios transmisores); se gún el tipo de contenidos, y simplificando un poco, el currículum transmitiría fundamentalmente contenidos instrumentales, mientras que el currí-

4. El concepto de metacomunicación puede definirse de varias maneras distintas. Verón (1970) en un artículo clásico e influyente acudió a la terminología de Jakobson hablando de [1] metacomunicación por selección y [2] metacomunicación por combinación, como una dimensión inherente a cualquier mensaje (todo lo que pudo ser seleccionado y no se seleccionó, etc.), identificándolo además a ideología. El término ha sido principalmente utilizado por Bateson (1971) y autores afines a su trabajo como [3] "el comentario sobre la comunicación", aunque también puede ser pensado, implicitando esta última idea, como [4] lo que se transmite relacionalmente (en la oposición clásica de Bateson, retomada por Watzlawick et al. 1967 como "contenido y relación"). A lo largo de este trabajo excluiremos el sentido [3], e intentaremos señalar a cuál de los otros corresponde el término cuando lo usemos. En cualquier caso, se trata de un "término-saco" que toma significados distintos en cada autor o en cada corriente teórica y cuya elucidación está lejos de haberse realizado. Quizás una posibilidad sería el considerarlo muy cercano a lo que se entiende por "tópic" en la semiótica textual (Eco, 1979), por más que sea sólo una traducción. 
culum oculto haría lo propio con contenidos expresivos ${ }^{5}$.

Debe entenderse: es imposible explicitarlo todo. Cuanto más se explicita, cuanto más se acerca un extremo del continuum, tanto más queda en la sombra por el otro lado. La analogía que proponemos es considerar que existe un límite a la explicitación, que hay una porción de currículum oculto que es revelable, pero que otra, no menos importante, permanece siempre oculta. Y ello es así por la propia naturaleza de la relación entre comunicación y metacomunicación: al explicitar algo más, algo nuevo, ésto conlleva su propio implícito que se suma al anterior, y así indefinidamente ${ }^{6}$. Esto es, desde luego, un inconveniente muy importante; pero también podemos sacar alguna enseñanza de él: por ejemplo, que pensar en explicitar el currículum como si se tratase de construir un metalenguaje de sus aspectos "ocultos" no lleva a ningún lado, o, mejor dicho, que lleva a cualquier lugar en el proceso indefinido de semiosis que caracteriza al lenguaje.

Este planteamiento nos conduce a enfatizar necesariamente el papel del currículum oculto, por más que sea un énfasis metodológico: ante un currículum determinado, sea considerándolo como realidad ya dada, sea en el proceso de su elaboración, la pregunta fundamental, antes incluso que la pregunta por las intenciones "ocultas", viene a ser: ¿qué se deja de enseñar?, ¿qué se transmite además de lo que se enseña? Sin embargo, estas preguntas no acaban de delimitar adecuadamente lo que hay que entender por metacomunicación, ni por tanto el principal sentido ce currículum oculto: la primera pregunta intenta indagar la metacomunicación por selección, pero es dudoso que metodológicamente sea correcto ya que no parte de un inventario claro sobre el que se efectúa la selección. La segunda pregunta intenta lo propio con la metacomunicación como relación (por oposición a contenido, cfr. nota 3), aunque es difícil pensar en aspectos relacionales desvinculados de contenidos.

\section{ANALISIS MODAL DEL CURRICULUM}

(3) Además creemos que se mezcla en estas discusiones otro eje de análisis: el que separa -o acerca- el currículum "escrito" (el currículum legal, lo que efectivamente figura como lo que debe ser señalado) del currículum "realizado" (lo que hacen los transmisores, las pequeñas o grandes modificaciones a las que someten al currículum: lo que es enseñado). $Y$ que alguna de las oposiciones anteriores se aplican a ambos extremos de este eje.

5. La dicotomía orden instrumental/orden expresivo ha sido utilizada en pedagogía por Bernstein (1966), por mas que luego no haya continuado en esta línea; en cualquier caso, el criterio de contenidos apuntado lo reservamos para lo que él denomina pedagogías invisibles (Bernstein, 1971).

6. Esta característica del lenguaje humano y de la comunicación en general ha sido puesta de manifiesto por numerosos pensadores. 
Incluso al punto intermedio, al que separa el currículum ideal, situado en el plano del deber ser, del currículum efectivo, de lo que hacen los agentes educativos; este punto podríamos representarlo por la asunción personal o/e institucional que hacen los agentes de aquellos aspectos, o de la totalidad en algunos casos, de ese currículum escrito no sea a su vez el producto de la transformación que unos determinados agentes y agencias han llevado a cabo. Tenemos, por tanto, al menos una doble transformación: a) la que se da para que el currículum escrito pueda existir $-\mathrm{T}_{1}-$; (b) la que llevan a cabo los agentes educativos "terminales" (maestros, padres, etc.) y que supone su asunción (o su crítica) personal del currículum $-\mathrm{T}_{2}-$.

Podrían señalarse asimismo otras dos transformaciones complementarias: la que se opera en los agentes educativos después de asumir un currículum determinado $-\mathrm{T}_{3}-$, y que les lleva a la adquisición de una competencia para poder llevarlo a cabo; y también la transformación final, la que separa este estado adquirido y la práctica de enseñar tal y como efectivamente la realizan los agentes $-\mathrm{T}_{4}$ -

$\mathrm{T}_{1}$ es aproximadamente lo que autores como Bourdieu denominan la arbitrariedad cultural, o, en general, el principio de análisis primero, y a veces único, de la denominada sociología del currículum: la selección de aquella parte de la cultura que se considera como importante para transmitir. Se trata, por tanto, de una dimensión esencial del estudio del currículum: considerar que éste sólo puede comprenderse por referencia a la selección que determinadas agencias sociales y educativas llevan a cabo de la totalidad de la cultura, esto es, por la definición de lo que se considera como cultura legítima, cultura dominante. De alguna manera esta selección es la más fundamental, la más ideológica", la que deja más partes ocultas que ninguna: es la selección que determina la positividad de lo enseñable.

De los aspectos que podríamos considerar, sólo vamos a detenernos en su estructura modal por ser el punto de vista que hemos elegido en este trabajo. Esta $\mathrm{T}_{1}$ se define como el paso de una modalidad del poder a una del deber. Más explícitamente: de todo lo que puede ser enseñado (el conjunto de la cultura) se selecciona una parte, aquello que debe ser enseñado. Debemos notar, sin embargo, que no es tan fácil: en ambos casos sólo plantearnos modalidades del hacer: poder hacer (o poder enseñar) y deber hacer (deber enseñar), pero no modalizaciones del ser: poder ser (poder ser enseñante de este currículum); las relaciones pueden complicarse más si las planteamos desde el punto de vista del alumno: deber aprender o/y poder aprender (este currículum), así como deber ser o/y poder ser alumno o "aprendiente" (de este currículum). Es evidente que incluso un análisis modal tan clásico y limitado desborda nuestros propósitos: sólo retendremos aquellos aspectos que directamente atañen la cuestión de las transformaciones apuntadas. 
El paso de una modalidad del poder a una del deber no es ni tan obvio ni ocurre sin consecuencias. Para empezar porque existen correspondencias señaladas entre ambas modalidades (Greimas y Courtès, 1979), pero también por tratarse de modalidades heterogéneas, no directamente mensurables a pesar de tales correspondencias (Brandt, 1985). Estas son, según Greimas y con denominaciones provisionales, las siguientes:

\section{CORRESPONDENCIAS ENTRE PODER Y DEBER Hacer}

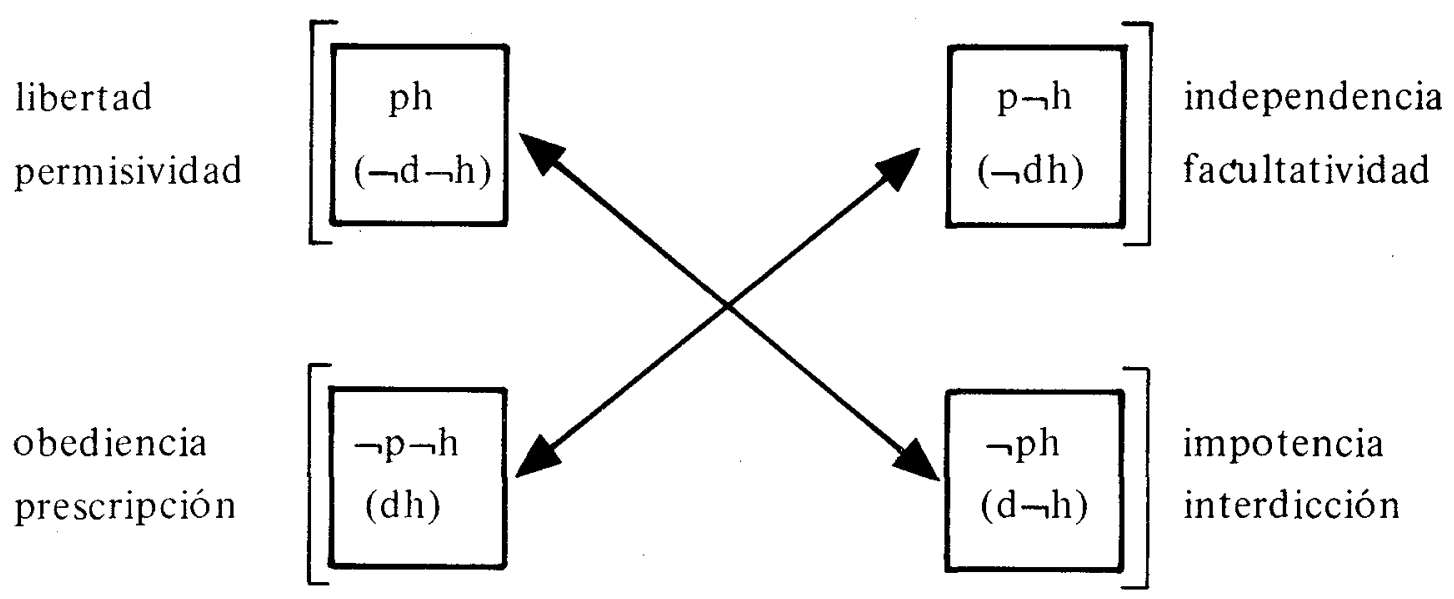

Y para el caso del poder y deber ser, el mismo Greimas establece las siguientes:

\section{CORRESPONDENCIAS ENTRE PODER Y DEBER Ser}

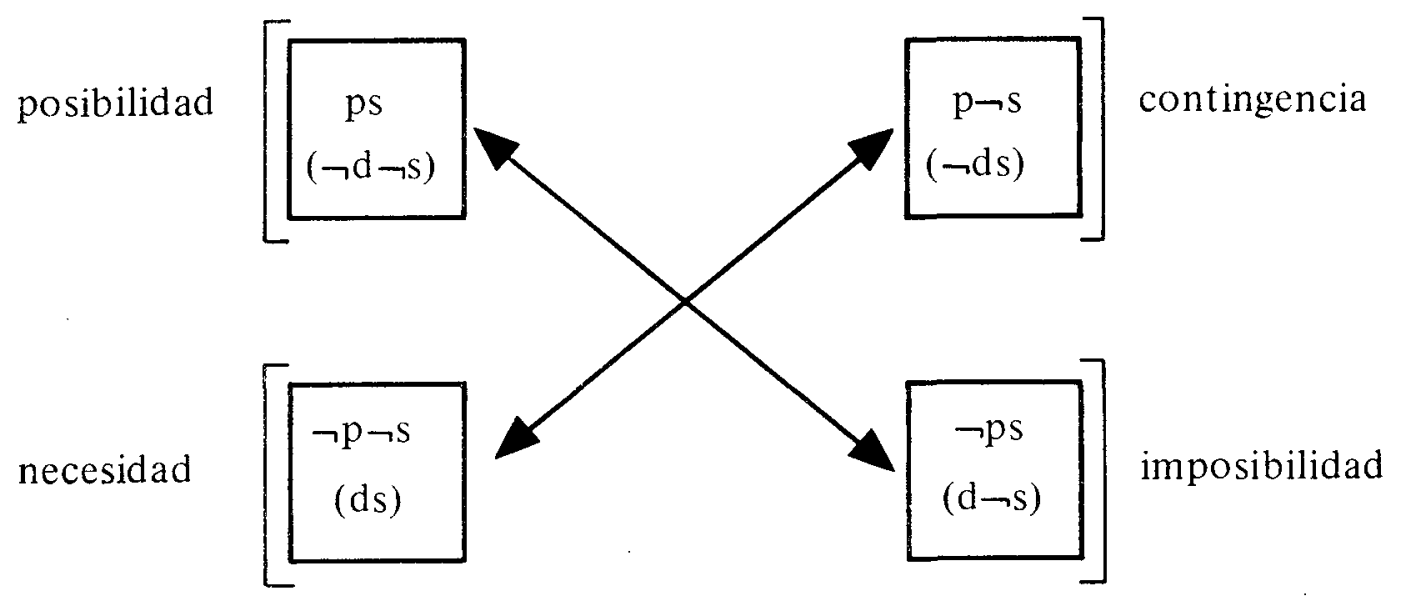

Donde, por comodidad en la notación, la expresiơn /-p -s/ se lee como "no poder ser". Se observará que en ambos casos hemos mantenido el cuadro original de /poder hacer/ y /poder ser/, acomodando las modalidades deónticas. Estas han sufrido una transformación consistente en ocupar el lugar correlativo en su respectiva deixis (así, ds ocupa el lugar de -d-s, y d-s 
ocupa el de -ds, etc $)^{7}$, esta $T_{1}$ se realizará por el paso siguiente: ph (-d-h) $\longrightarrow \mathrm{dh}(-\mathrm{p}-\mathrm{h})$. Obviamente esta construcción se lleva a cabo mediante el paso previo por la deixis negativa (asegurando primero una negación contradictoria): $\mathrm{ph}(-\mathrm{d}-\mathrm{h}) \longrightarrow-\mathrm{ph}(\mathrm{d}-\mathrm{h}) \longrightarrow-\mathrm{p}-\mathrm{h}(\mathrm{dh})$. Esto es, la $\mathrm{T}_{1}$ "guarda memoria" del paso por [-ph (d-h)]: no sólo se pasa de la pura posibilidad a la necesidad (o de la libertad a la prescripción), sino que simultáneamente se define implícitamente lo que es imposible (o lo que está interdicto), dejando el reino de lo contingente (o de lo facultativo) fuera de su ámbito.

Esta es quizás una primera aproximación fuerte al concepto de currículum/currículum oculto: se "legisla" lo que debe hacerse (como una parte de lo que puede hacerse o de lo que no debe no hacerse), a la vez que se excluye lo que debe no hacerse, pero no se impone lo que no debe hacerse. De esta ambigüedad entre lo que debe no enseñarse y lo que no debe enseñarse es de donde creemos surgen algunas dificultades para retrazar lo "oculto" del currículum. El margen de lo que no debe hacerse puede variar en cada pedagogía: desde casi no existir - como es el caso de las pedagogías autoritarias, que son tales no sólo por la imposición de un deber hacer más un deber no hacer sino también y sobre todo por un casi nulo no deber hacer-, hasta ser casi tan amplio como el propio deber hacer.

De alguna manera, las modalidades deónticas incluyen la existencia de un sujeto presupuesto, de un destinador, que obliga a un deber, a una cierta imposición, para el sujeto modalizado, una coerción en su hacer, que no está presente en las modalidades aléticas (esta transformación de lo alético en lo deótico se podría también nombrar -como sin duda preferirían Bateson, Wilden y otros- como la digitilización de un contínuo, o la discretización de lo análogico). Desde el punto de vista de los actores sociales implicados en esta transformación primera, lo cierto es que en el plano idealizado (pero real) del currículum en el que nos movemos esta primera transformación se lleva a cabo por una suerte de actante colectivo e impersonal y que es nombrado genéricamente ("comisiones" para elaborar planes de estudios, "el Ministerio", etc.), sobre cuyo pseudoanonimato recae la falta de asignación de sentido que los restantes actores y actantes del proceso suelen adjudicar al resultado de la transformación. Sin embargo, los actores pueden ser múltiples $-\mathrm{y}$ de hecho lo son: los autores de libros de texto, por ejemplo, efectúan una actualización de los principios y temas (objetos de

7. La terminología greimasiana que utilizamos puede verse en Greimas y Courtès (1979). Sobre el cuadrado semiótico, además del citado diccionario, Greimas y Rastier (1968) por ser el artículo princeps. Aspectos técnicos sobre el cuadrado pueden encontrarse en Nef (comp, 1976), Utaker (1974), Petitot (1977), así como en el monográfico dedicado por Actes Sémiotiques. Le Bulletin. Un análisis distinto, que intenta superar algunos de los problemas que la modelización mediante el cuadrado semiótico conlleva, es el realizado por Brandt $(1984,1985)$. En cuanto a la teoría semiótica de las modalidades, algunos de los principales trabajos son: Greimas (1976a, 1976b, 1983), Greimas y Landowski (comps, 1979), Darrault (comp, 1976), Coquet (1985), etc. 
valor, en la terminología que utilizamos), o bien el propio grupo de profesores de una escuela, cada profesor por separado, etc.

$\mathrm{T}_{2}$ es la transformación por la que los sujetos asumen (o no) el currículum. Modalmente es el paso de una modalidad del deber (en tanto que un deber exterior al sujeto) a una del querer - querer hacer, querer enseñar. Se trata, obviamente, de la culminación de una fase del esquema narrativo: el contrato. Si la $\mathrm{T}_{1}$ aseguraba la existencia de un destinador y la configuración de un deber hacer y de un deber ser para el destinatario, ésto es, un hacer persuasivo por parte del destinador (o de las figuras que tomasen a su cargo tal tarea), la $\mathrm{T}_{2}$ por el contrario representa el hacer interpretativo del destinatario, el juzgar sobre la fiducidad del contrato, incluso llevar a cabo un juicio veridictorio sobre el mismo y, en consecuencia, aceptar o no el programa narrativo que el contrato conlleva.

El destinador puede ejercer una persuasión fuerte-constante sobre la aceptación del contrato, acompañada de otros haceres persuasivos en otros programas narrativos que se entremezclan con el del currículum. Esto es, la asunción del deber hacer curricular por parte del sujeto modalizado ( $\mathrm{y}$ modalizador), del destinatario, no es necesariamente un hacer interpretativo ejercido "libremente" (sobre esta cuestión, la inserción del currículum en estructuras educativas más amplias, volveremos más adelante), por lo que es difícil intentar establecer una combinatoria de la multiplicidad de programas narrativos/contratos a los que el destinatario tiene que hacer frente simultáneamente. Ahora bien, el destinatario sólo puede optar lógicamente entre tres alternativas con algunas variantes:

(1) aceptar completamente el currículum propuesto:

(1a) llevarlo a cabo,

(1b) no llevarlo a cabo.

(2) rechazar completamente el currículum propuesto:

(2a) proponer un currículum alternativo,

(2b) no proponer un currículum alternativo.

(3) modificar parcialmente el currículum propuesto.

Estas tres alternativas configuran los posibles resultados $T_{2}$, aunque no el proceso que constituye la transformación. Su caracterización modal podría ser la siguiente:

1a. $d h+q h(d h)+h(d h)$

1b. $d h+q h(d h)+-h(d h)$

2a. $d h+-q h(d h)+q h(d h 2)$

2b. $d h+-q h(d h)+q-h$

3. $\mathrm{dh}+\mathrm{qh}(\mathrm{dh} 3)$

Donde $\mathrm{dh}_{2}$ se define como: $\mathrm{dh}_{2} \wedge \mathrm{dh}=\mathbb{S}$, y donde $\mathrm{dh}_{3}$ es una reformulación de $\mathrm{dh}$, esto es, integra partes de dh como programa narrativo de uso del nuevo programa narrativo de base, sin poder decidir a priori su número 
y colocación. Como se ve, la modalización volitiva final del sujeto es distinta a cada caso (y, asimismo, sus relaciones con el deber ser), aunque la lexicalización de estas modalidades está lejos de poderse establecer mínimamente y, por tanto, es difícil "dar nombre" a estas posiciones.

$\mathrm{T}_{3}$ es una transformación que consiste en la adquisición de un determinado saber. Se trata de la transformación modalizadora habitual: el sujeto se vuelve competente para poder llevar a cabo la performance que requiere el programa narrativo asumido. La hemos planteado como dependiente del querer hacer, pero es obvio que hay otras posibilidades: quizás sea suficiente con señalar que son adquisiciones modales en muchos casos interdependientes. Dos casos nos parecen importantes: aquél en el que el querer es previo, está contemplado como una fase del contrato por parte del destinatario -y que es en el que nos hemos movido hasta ahora-, y otro en que el saber hacer del destinatario es anterior a su aceptación del deber hacer sugerido por el destinador. Este último plantea la problemática de considerar al destinatario influído por su competencia ya adquirida y reformular así el contrato en función de ella. Decidir cuándo se efectúa así, sólo es posible al analizar discursos ocurrencia y no al intentar retrazar el esquema narrativo del paso (idealizado) del currículum escrito al currículum realizado.

Por otra parte, esta transformación puede no realizarse: en un buen número de casos el sujeto modalizado por el querer hacer posee ya la competencia modal del saber hacer necesaria para llevar a término el programa narrativo asumido. $\mathrm{O}$, como mínimo, posee en muchos casos lo que se denomina metasaber, esto es una competencia sobre el saber hacer: el saber saber hacer.

Finalmente, hemos también nombrado una última transformación $\mathrm{T}_{4}$, cuyo resultado sería el enseñar, el hacer. Es una transformación que en la terminología semiótica que venimos utilizando se corresponde con la performance (Greimas y Courtès, 1979). En ella el sujeto modalizado según un querer hacer (un deber hacer asumido) y un saber hacer, lleva a término un cambio de estado de la relación que otro sujeto mantiene con un objeto. El sujeto se ha transformado en sujeto operador (o sujeto modalizador, según sea la naturaleza del objetivo: valor o modal) que actúa sobre un sujeto de estado, modificando su relación con el objeto. La performance ha sido muy estudiada por los semióticos, aunque las nociones primitivas que se utilizan (sujeto, objeto, acción, etc.) todavía van a permitir un análisis semiótico más detallado ${ }^{8}$. Pero aquí nos interesa analizar esta transformación

8. Como sugieren las conexiones entre semiótica e inteligencia artificial, por ejemplo, y las concepciones sobre la representación del conocimiento que se encuentran en la base de ambas disciplinas (Desclès, 1985). Otras posiciones sobre el problema son las que provienen de la teoría recientemente formulada por Stöckinger $(1985,1986)$ y de la teoría de catástrofes (Petitot, 1986). La problemática, que consideramos crucial para la semiótica y las ciencias cognitivas, excede con mucho los intereses de este trabajo. Sin embargo, es obvio su interés para la reflexión pedagógica dada la importancia que las concepciones sobre la acción educativa han ido adquiriendo en los ultimos años. 
en relación al currículum.

En efecto, esta transformación última la habíamos hecho equivaler a la idea de currículum realizado, del currículum en tanto que práctica (o mejor, relación) educativa. Esta acepción de currículum es inusual, aunque para nosotros ha tenido un gran valor expositivo: situada en un extremo del proceso, nos ha permitido entender cómo existe una continuidad (para la figura del maestro que hemos analizado) entre el currículum escrito y el acto concreto de enseñar -y ello sin necesidad de entrar constantemente en el análisis de las agencias educativas implicadas: desde la familia, pasando por la escuela, hasta el conjunto de agencias sociales encargadas de "transformar" el currículum, como las editoriales de libros de textos, o las divulgaciones científicas en periodicos, revistas o televisión, etc.-. Pero seguir pensando en el currículum realizado como un concepto fuerte nos parece que es forzar la analogía inicial: su estudio, el del acto de enseñar, queda un poco alejado del estudio del currículum, se mantiene como un límite del mismo, pero como un aspecto central -al menos de este trabajo-.

El siguiente cuadro resume un poco el sentido general de las distinciones realizadas y los ejes sobre los que se sitúan, así como las diversas transformaciones implicadas:

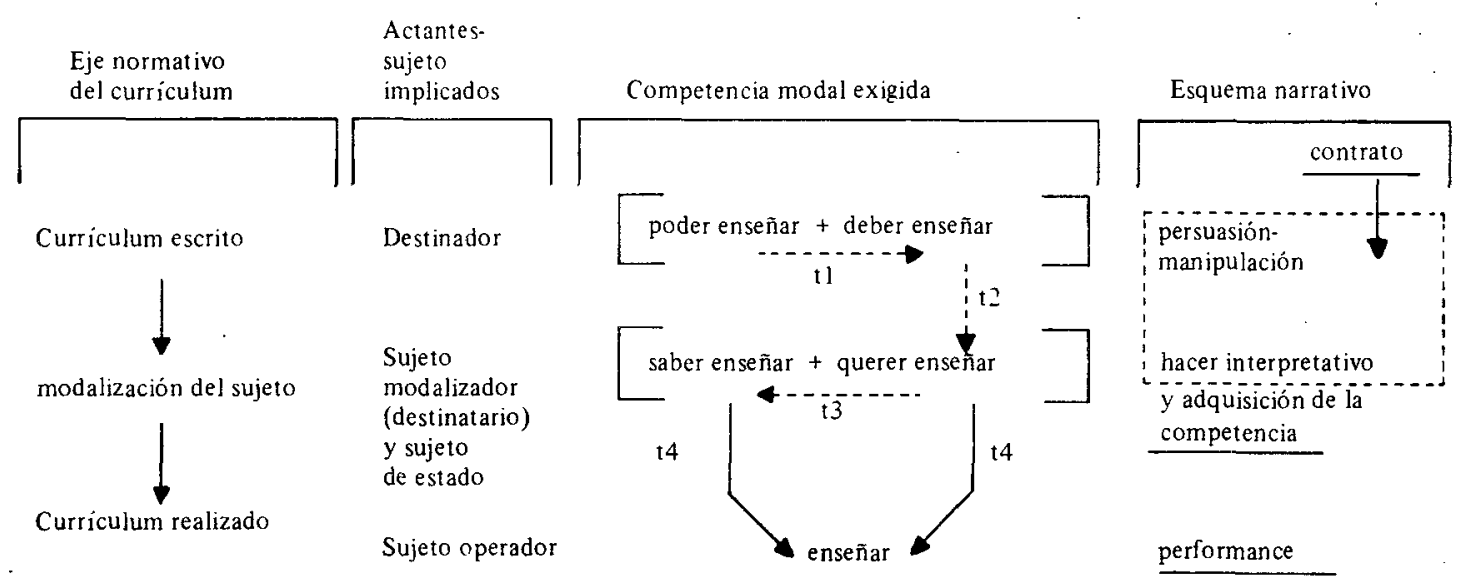

\section{EL LUGAR DEL CURRICULUM EN UNA TEORIA DE LA EDUCACIÓN}

Hasta ahora hemos llevado a cabo dos análisis distintos, y esperamos que complementarios, sobre el curriculum: (1) su relación con el currículum oculto y el esclarecimiento que aporta el concepto de metacomunicación; (2) un bosquejo de análisis modal y narrativo de las diferentes transformaciones y actantes implicados en el paso del currículum escrito a la práctica eductiva concreta.

Creemos, por otra parte haber mostrado cómo un mismo concepto puede 
implicar diversas disciplinas o "ciencias de la educación": $\mathrm{T}_{1}$ parece ser el tema de análisis de la mayoría de los trabajos de sociología de la educación; $\mathrm{T}_{4}$ es objeto claro de la didáctica. $\mathrm{T}_{2} \mathrm{y} \mathrm{T}_{3}$ se encuentran en tierra de nadie, o en tierra de todos según se quiera. Es obvio que esta repartición de puntos de vista no tienen porqué ser aceptada por los investigadores que trabajan en el interior de la didáctica, o de la sociología de la educación, o de la psicología de la educación; pero también es verdad que responde a criterios de trabajo previo sobre el tema, ya históricos, que difícilmente pueden ser negados. La importancia de señalar los diferentes niveles de análisis implicados ante cualquier concepto de una teoría de la educación radica en intentar demostrar su interconexión, en cómo cada análisis particular de un concepto puede (y quizás debe) ser completado con los otros niveles de uso de los conceptos educativos ${ }^{9}$, y sobre todo en lograr la continuidad lógica entre los diferentes análisis antes incluso que en intentar una teoría global completa, en todos los niveles de uso.

Sin embargo, no podemos pretender haber acabado la tarea descriptiva del currículum. Al contrario, no sólo el análisis efectuado ha sido fragmentario y esquemático, sino que ha partido de la consideración formal del mismo como requisito inicial; esta formalidad suponía por ejemplo, atender sólo a aspectos generales, hablar del currículum como entidad única, y correlativamente no entrar en analizar los distintos tipos de currícula existentes. Esta cuestión, la clasificación de los currícula, responde a un requisito central en cualquier teoría de la educación, al menos al componente descriptivo de la misma: no limitarlo a una regla definitoria o de reconocimiento -tarea bien compleja por demás-, cuanto intentar demostrar que los criterios fenomenológicos en los que se basa esta capacidad de distinción responden a una distinta organización.

Bernstein distinguió, hace ahora veinte años, dos tipos fundamentales de currícula, el integrado y el "apilado" o "compilado". Sus diferencias respondían a cómo estaba organizada la relación entre las materias que componían el currículum. La mezcla de categorías generaba el currículum integrado, y la pureza - por usar el término de Mary Douglas que se halla en la base de la distinción, Douglas, 1966- generaba por el contrario reglas de aislamiento entre categorías, currícula apilados. Esta distinción es formal; luego, y permite por ello mismo ver similitudes en currícula aparentemente cercanos. Prototípicamente, el principio explicativo de los diferentes ti-

9. Ya nos referimos a nuestra concepción de los niveles de uso del término educación. Bastará señalar la distribución de las distintas transformaciones en función de los mencionados niveles y sólo desde un punto de vista analítico: $\mathrm{T}_{1} \longrightarrow$ nivel social; $\mathrm{T}_{2}$ y $\mathrm{T}_{3} \longrightarrow$ nivel organizativo; $\mathrm{T}_{4} \longrightarrow$ nivel interaccional. El nivel biológico-cognitivo sólo puede plantearse como los efectos del currículum sobre los sujetos, dimensión que no hemos considerado pero que está presente en muchos trabajos sobre el currículum (los que intentan coordinar varios niveles de análisis, como por ejemplo Dreeben, 1976). 
pos de currícula - en cuanto currícula ocurrencia - es la "clasificación"10, en el sentido bernsteiniano del término, esto es la fuerza de la separación entre contenidos o categorías. La "clasificación" es la operación objetivada equivalente a la transformación primera que habíamos comentado $\mathrm{T}_{1}$ : la transformación de la aparentemente pura posibilidad a una organización concreta presentada como real (lo que es, como si fuese lo único posible, lo que debe ser). En este sentido, podrían redefinirse los dos tipos de "clasificación" encontrados por Bernstein (fuerte y débil) como:

$\mathrm{C}^{+}$(máxima fuerza de la barrera de separación, categorías claramente separadas $)=\mathrm{ds}\left(\mathrm{S} \backslash / \mathrm{O}_{1}, \vee \mathrm{O}_{2}, \ldots \mathrm{O}_{\mathrm{n}}\right)$.

$\mathrm{C}^{-}$(mínima fuerza de la barrera de separación, categorías mezcladas $)=$ ds $\left(\mathrm{S} \vee \mathrm{O}_{\mathrm{c}}\right)$. Donde $\mathrm{O}_{\mathrm{c}}$ representa un objeto completo, esto es, un objeto que no es un simple agregado de objetos parciales.

Se entenderá que la distinción de Bernstein reposa sobre la naturaleza del objeto valor o/y modal antes que sobre la modalización sufrida por el sujeto por parte del destinador. En ambos tipos de "clasificación", la modalización es el resultado de la $\mathrm{T}_{1}$, por mas que la hayamos planteado sólo como un deber ser. Lo que nos parece importante es que (los objetos y) la relación de estado consiguiente es siempre una relación actualizada, que sólo se realizará en la performance. Ello permite entender un aspecto particularmente difícil: el "sentido de realidad" que la organización del currículum adopta. Parece como si una institución escolar fuertemente clasificada definiera completamente el tipo de relaciones educativas que en ella pueden darse, el currículum compilado, los roles de profesor y alumno, etc. (Bernstein, 1967); evidentemente es así pero también es verdad que las instituciones educativas no son estáticas - aunque tengan un ritmo de cambio muy lento-, y que debe contemplarse la posibilidad de que cambien incluso los principios de "clasificación" que las definen. La alternativa para ello es considerar lo que Bernstein denomina enmarque (inglés, framing), y que nosotros pensamos como las variaciones "discursivas" que acontecen especialmente en la performance, en el acto concreto de enseñar; pero, para ello, es igualmente necesario plantear que la relación con los objetos que genera la "clasificación" es sólo actual y no realizada".

La clasificación de los currícula adoptada por Bernstein (mediante el concepto de "clasificación") merece además otro comentario. Pues el criterio en que se fundamenta permite entender, o rehacer, la noción de currículum oculto. En efecto, la "clasificación" tiene una doble lectura en la obra de este gran teórico: bien como realidad "objetiva" (en el sentido de escuelas abiertas y cerradas, la clasificación del espacio y del tiempo, etc,) bien

10. Entrecomillaremos / clasificación / para referirnos al concepto bernsteniano, dejando el término normal para su uso no técnico.

11. De ahí la relación disjuntiva del sujeto con el objeto en la definición del enunciado de estado. 
como realidad percibida, como cognición del sujeto que interpreta fuerte o débilmente clasificado un determinado contexto experiencial (paradigmáticamente en el trabajo de Holland 1979 y en Bernstein 1986), esto es, como ground rule o recognition rule (para la terminología, Bernstein 1981, 1985).

Esta aparente ambigüedad del concepto se resuelve, pero no mediante una antinomía objeto-subjetivo, sino pensando que es el propio proceso de transmisión cultural el encargado de llevar a cabo la "in-corporación por el sujeto de la organización social de la realidad (de nuevo, la figura de la banda de Moebius...), vía los procesos de aprendizaje. Sin embargo, el núcleo de la tesis de Bernstein es que tal proceso no es "culturalmente indiferenciado", esto es, que no se produce igual para todos lo sujetos, sino en función del lugar que ocupan en la estructura social. La idea es, pues, la de la desigualdad en el origen familiar-cultural-de clase), que provoca afectos diferenciales incluso ante una misma situación (la escuela, y, en general, las agencias de socialización secundarias). Por tanto, la "clasificación" de la "realidad", de la realidad tal y como aparece fenomenológicamente: del estado de las cosas"'(Sachverhalte), es algo que existe "realmente" (valga la redundancia socialmente - por más que sólo pueda ser conceptualizada a través de un observador dotado de lenguaje y en una posición social determinada. Esta existencia real de la "clasificación" se produce siempre en el interior de relaciones de poder o/y de control 12 .

Quizás conviniese resaltar todavía más hasta qué punto los conceptos bernsteinianos son plenamente estructurales (y ello en el doble sentido de formales y dinámicos), para comprender así su clasificación de los currícula -que, aparentemente, es sólo formal [sin embargo, Thompson (1981) y, para una presentación, Rodríguez Illera (1983)]. En efecto, la vieja tesis de Durkheim y Mauss (1903) sobre el origen social de la clasificación en que se basan los sistemas de conocimiento - y que es, dicho sea de paso, uno de los fundamentos de la sociología del conocimiento- ha sido criticada e ignorada a la vez (Bloor, 1982, para un resumen de la polémica y para un intento de superarla), y probablemente necesita correcciones importantes en la forma como fue enunciada; sin embargo, está en el mismo núcleo de la teoría de los códigos y de todo el tratamiento de la "nueva sociología de la educación" ha hecho del currículum. La importancia de los dos tipos de currícula, y de sus relaciones, radica probablemente en mostrar como surgen y cómo se integran en estructuras más amplias, los códigos educativos, que siguen siendo el resultado de la combinatoria de las diferentes "clasificaciones" y enmarques.

12. Quizás una lectura esquemática de Bernstein (especialmente, 1981, 1985) podría sugerir que la clasificación depende sólo de las relaciones de poder. 


\section{DISCUSION}

Se observará que a lo largo de este trabajo hemos intentado desarrollar (en esquema) tres argumentos distintos sobre el currículum:

1) la comprensión del denominado currículum oculto mediante la puesta en paralelo con el concepto de metacomunicación; se trata más de una idea exploratoria que de una verdadera comprensión, pero al menos ha servido para señalar lo obvio: que tanto currículum como currículum oculto son auténticos sacos conceptuales y que en torno a ellos se teje un nudo de problemas centrales para la teoría de la educación. Pero que depende de cómo se enfoquen, de qué se intente demostrar, de cuál sea la pregunta en definitiva, para que la teoría del currículum responda una cosa u otra, o no responda nada en absoluto.

Trazar la polisemia de esta pareja de términos es tarea a realizar y que permitirá, al menos, empezar a delimitar los distintos usos que hoy en día aparecen en los varios contextos teóricos que los utilizan. Algo de ello hemos pretendido, al distinguir entre el currículum escrito y el currículum realizado.

2) si el currículum tiene alguna realidad y no sólo teórica sino experiencial -individual y social-, por más que no se lo nombre necesariamente como tal por los actores implicados, el problema que se plantea es saber cómo los mencionados actores lo significan, lo subjetivizan. Esta doble cuestión, la significación social e individual de currículum, es la que hemos tratado al plantear el análisis semiótico. Distinguir los diferentes tipos de transformaciones asociados, e intentar caracterizar modalmente las distintas posiciones del actante-sujeto (individual o colectivo) a ellas asociado, ha sido lo que hemos bosquejado.

3) explicitar el interés de nuestro trabajo para la teoría de la educación, así como exponer algunas de las ideas de Bernstein ha permitido relacionar "clasificación" y currículum. Sin embargo, la línea que une currículum oculto, metacomunicación, análisis modal del mismo y la problemática de la "clasificación" sólo ha podido empezar a señalarse. Una tarea de análisis conceptual de todas estas nociones es quizá la tarea más urgente para poder empezar a hablar con rigurosidad de estos problemas. 


\section{BIBLIOGRAFIA}

ARACIL, Y. (1982): Papers de sociolinguistica. Barcelona, La Magrana, 1982.

ARONOWITZ, y GIROUX, H (1985): Education under siege

BATESON, G. (1971): Steps to an ecology of mind. New York, Ballantine, 1972.

BERNSTEIN, B. (1966): "Sources of disaffection and consensus in education", en B. Bernstein (1977), pp. 37-49.

BERNSTEIN, B. (1966): “On the curriculum", en B. Bernstein (1977), pp. 79-84.

BENRSTEIN, B. (1967): “Open schools, open society?”, en B. Bernstein (1977), pp. 67-75.

BERNSTEIN, B. (1977): Class, Codes and Control, Vol. 3. Towards a Theory of Educational Transmissions. London, Routledge and Kegan, 1977, 2a ed.

BERNSTEIN, B. (1981): "Codes, modalities and the Proces of Cultural Reproductión", en M. Apple (comp.) Cultural and Economic Reproduction in Education. London, Routledge, 1982).

BENRSTEIN, B. (1985): “On Pedagogic Discourse". No publicado.

BLOOR, D. (1982): "Durkheim and Mauss Revisited: Clasification and the Sociology of Kworeledge", Stud. Hist. Phil. Sci.., vol. 13, 4, pp.267-297, 1982.

BORDIEU, P. (1982): Ce que parler veut dire Paris, Fayard, 1982.

BRANDT, P.A. (1984): "Fragments d'une analyse modale de l'éthique aristotélicienne", Actes Sémiotiques. Bulletín, VII n. 31, 1984. También en P.A. Brandt: Recherches Sémiotiques (1971-1984), Poetica et Analytica n. 1985, pp. 569-577, Aarhus.

BRANDT, P.A. (1985) "Aléthique et déontique. Esquisse d'une nouvelle analyse sémiotique des modalités", H. Parret y H. G. Ruprecht (comps): Exigences et perspectives de la sémiottique, vol. 1., pp. 123-134, Benjamins.

COQUET, J. (1985): Le sujet et son discours. Vol. 1. Essai de grammaire modales. Paris Klincksieck, 1985.

DARRAULT. I. (1976, comp.): Modalités. Logique, linguistique, sémiotique. Langages, 43, 1976, Paris.

DESCLES, J.P. (1985): “Representatión des connaissances”, Actes Sémiotiques, Documents, VII n. 69-70, 1985.

DOUGLAS, M. (1966): Pureza y peligro, Un análisis de los conceptos de contaminación y tabú. Madrid, S. XXI, 1973.

DREEBEN, R. (1976): "El currículum no escrito y su relación con los valores". En J. Gimeno Sacristán y A. Pérez Gómez (1983, comps): pp. 73-85.

DURKHEIM, E. y Mauss, E. (1903): "De quelques formes primitives, de classification", en M. Mauss: Essais de sociologie, París, Seuil, pp. 162-231, 1971.

ECO, U. (1979): Lector in Fabula. Barcelona, Lumen, 1981.

GIMENO SACRISTAN, J. y Pérez Gómez, A. (1983, comps.): La enseñanza: su teoría y su práctica. Madrid, Akal, 1983.

GREIMAS, A.J. (1976a): "Pour une théorie des modalités", en Langages, 43, 90-107. Reimpreso en Greimas (1983), pp. 67-91.

GREIMAS, A.J. (1976b): Maupassant. La sémiotique du texte: exercices pratiques. Paris, Seuil, 1976. [v. cast. ed. Paidós]

GREIMAS, A.J. (1983): Du Sens II. Paris, 1983.

GREIMAS, A.J. y COURTES, J. (1979): Sémiotique Paris, Hachette, 1979. [v. cast. ed. Gredos]

GREIMAS, A.J. y COURTES, J. (1986, comps): Sémiotique, Vol. 2. Paris, Hachette, 1986.

GREIMAS, A.J. y LANDOWSKI, E. (1979): Introduction à l'analyse du discours en sciences sociales. Paris, Hachette, 1979.

GREIMAS, A.J. y RASTIER, F. (1986): "Las reglas del juego semiótico", en A.J. Greimas: En torno al sentido. Madrid, Fragua, 1973, pp. 153-183.

HOLLAND, J. (1979): “La orientación social al significado", Infancia y Aprendizaje, 7, 1979. 
NEF, F. (1976, comp): Structures elémentaires de la signification. Bruxelles, Complexe, 1976. PÊCHEUX, M. (1969) Hacia el análisis automático del discurso. Madrid, Gredos, 1978.

PETITOT, J. (1977): “Topologie du carré sémiotique”, Etudes Littéraires, vol. 10,3,347-428, 1977, Laval.

PETITOT, J. (1986): Artículos contenidos en Greimas y Courtès (1986, comps.).

RODRÍGUEZ ILLERA, J.L. (1982): "Requisitos de una teoría de la educación", II Seminario de Teoría de la Educación, Salamanca, 1982.

RODRÍGUEZ ILLERA, J.L. (1983): "Currículum, control y catástrofes”, Documentos de Tecnología educativa, Madrid, 1, pp.

RODRÍGUEZ ILLERA, J.L. (1985): Teoría de la educación y de la socialización. Tesis Doctoral, Universidad de Barcelona, 1985.

STÖCKINGER, P. (1985): "Prolégomènes à une théorie de l'action", Actes Sémiotiques. Documents, VII, 62, 1985.

STÖCKINGER, P. (1986): Artículos contenidos en Greimas y Courtès (1986, comps).

THOMPSON, M. (1981): Rubbish Theory. Cambridge, Cambridge University Press, 1981.

VÁZQUEZ, G. (1985): "Currículum oculto y manifiesto", en VV.AA.: Condicionamientos socio-políticos de la educación. Barcelona, Ceac, 1985, pp. 169-187.

YERÓN, E. (1970): "Ideología y comunicación de masas", en E. Yerón (comp.): Lenguaje $y$ comunicación social. Buenos Aires, Nueva Visión, 1970.

WATZLAWICK, P. et al. (1967): Teoría de la comunicación bumana. Buenos Aires, Tiempo Contemporáneo, 1969, y ed. Herder, Barcelona. 
